\title{
Comparing Different Accelerometer Cut-Points for Sedentary Time in Children
}

\author{
Claudia Fischer and Mine Yıldırım* \\ VU University Medical Center
}

\author{
Jo Salmon \\ Deakin University \\ Mai J.M. Chinapaw \\ VU University Medical Center
}

*Claudia Fischer and Mine Yılıdrım share the first authorship.

\begin{abstract}
Actigraph accelerometers are hypothesized to be valid measurements for assessing children's sedentary time. However, there is considerable variation in accelerometer cut-points used. Therefore, we compared the most common accelerometer sedentary cut-points of children performing sedentary behaviors. Actigraph Actitrainer uniaxial accelerometers were used to measure children's activity intensity (29 children, 5-11 years old) during different activities, namely playing computer games, nonelectronic sedentary games, watching television and playing outdoors. A structured protocol was the criterion for assessing the validity of four common cut-points $(100,300,800,1100$ counts/minute). The median counts during all sedentary behaviors were below the lowest comparison cut-point of $100 \mathrm{cpm}$. The 75th percentile values for the sedentary behaviors were always below the cut-point of $300 \mathrm{cpm}$. Our results suggest that the cut-point of $<100 \mathrm{cpm}$ is the most appropriate.
\end{abstract}

It has been argued that recent increases in overweight and obesity prevalence in youth (22) can partly be ascribed to the increased time spent sedentary (8). Sedentary behaviors is marked by a low metabolic equivalent intensity level (2) that involves activities primarily performed while sitting (e.g., television viewing, using the computer, playing electronic games). While secular trends suggest that there have been few changes to the amount of daily television viewed by young people in the last 50 years, access to electronic entertainment media in the home

Fischer, Yıldırım, and Chinapaw are with the EMGO Institute for Health and Care Research and Dept. of Public and Occupational Health, VU University Medical Center, Amsterdam, The Netherlands. Salmon is with the Centre for Physical Activity and Nutrition Research, School of Exercise and Nutrition Sciences, Deakin University, Melbourne, Australia. 
has increased substantially, and it has been estimated that engagement with these media accounts for up to five hours of leisure-time among youth each day (10). The most common forms of screen-based sedentary behaviors among children are television watching and computer use (5). It is known that one can be highly active, meeting the physical activity guidelines, and at the same time highly sedentary (19). Sedentary behavior should be investigated as a separate and unique construct due to its independent health effects, determinants and related biological processes (20). A recent review showed that there is an inverse longitudinal relationship between sedentary behavior and aerobic fitness levels of children (6). Further the review showed that increased sedentary time was associated with higher cardiovascular risk, insulin resistance and higher risk of obesity among adolescents (9) (11).

Valid and reliable methods for assessing sedentary behaviors are crucial for public health strategies and preventive purposes in the area of childhood obesity. For example, the validity of most survey measures of television viewing is of unknown quality (4). Therefore, where possible, the use of objective measures of sedentary behaviors is preferable (16). Also progressively more governments propose a maximum screen time for children of 2 hours per day (e.g., UK, Canada, Australia) which emphasizes the need for the designation of a "common" sedentary cut-point.

Accelerometers have been frequently used as an objective measure of physical activity, which assesses the amount and intensity of physical activity by motion sensing (7). However, accelerometers are also emerging as a potentially useful method for assessing children's sedentary time (16).

Among children, several different cut-points of accelerometer movement counts from the vertical axis of the Actigraph accelerometer (the most commonly used accelerometer device in the physical activity field; 16) have been proposed for defining time spent as sedentary. Unfortunately, there is no consensus on cut-point values resulting in a large diversity between researchers. For example, Alhassan et al. applied $\leq 1592$ counts per minute (cpm) as a cut-point for five year old children (3). Sardinha et al. Compromised between the lowest and highest published sedentary cut-points and used $<500 \mathrm{cpm}$ as a threshold (17). Using US prevalence data, Matthews et al. applied a cut-point of $<100 \mathrm{cpm}$ to estimate time spent sedentary across all age groups (from six years), a value often used to define sedentary time among adults (12), but previously validated for adolescent girls by Treuth et al. (21).

Some cut-points are derived from observational studies with young children (3-4 years; 15) and slightly older children (8-10 years; 18$)$, and others from calibration studies using whole room calorimetry with 6-16 year olds (14) or using a portable metabolic unit with 13-14 year old girls (21). Each of these studies has developed a unique cut-point for estimating young people's sedentary time, and as illustrated by Reilly, when these various cut-points are compared within a single sample, large differences in estimates of sedentary time can result (16). With so many cut-points available, the field of sedentary behaviors does not need yet another published cut-point (23). However, there may be value in examining how these existing cut-points compare when children engage in a number of different sedentary behaviors.

The aim of this study was to compare and identify the most appropriate accelerometer cut-point for a number of different sedentary behaviors (i.e. playing computer games, nonelectronic sedentary games, watching television) in children using a structured protocol as the criterion measure. We evaluated the validity of four commonly used cut-points which were derived from calibration studies namely 
$100 \mathrm{cpm}$ by Treuth et al. (21), $300 \mathrm{cpm}$ by Stone et al. (18), $800 \mathrm{cpm}$ by Puyau et al. (14) and $1100 \mathrm{cpm}$ by Reilly et al. (15).

\section{Methods}

\section{Participants}

In the current study, a convenience sample of 29 apparently healthy children aged 5 - to 11 -years old ( $28 \%$ boys) were recruited. Data from four children were excluded from the final analysis due to inappropriate accelerometer data processing. Children were recruited from primary schools in the neighborhood of the VU University Amsterdam and from a child day care center in Austria. Children and their families were informed beforehand about this study and gave their verbal consent.

\section{Structured Protocol and Data Collection}

Each of the following supervised activities were performed for a 20 -min period: children played electronic games on a computer while sitting on a chair; children played with a variety of sedentary games involving small movements (cards (UNO), jacks, puzzles, Lego, miniature cars, Mikado, excluding exergames) on a floor mat; children watched television while sitting on a chair or couch; and children played outside their house or at a playground which included running around, playing, and tapping with a ball (they were not instructed to move continuously to more accurately reflect their natural movement patterns). The first three activities reflected common sedentary behaviorss that children engage in, while "outdoor playing" was also included to determine whether the various sedentary cut-points could distinguish between children's sedentary behaviors and higher intensity active free play.

Children were asked to wear an ActiGraph-Actitrainer research model accelerometer (ActiGraph, LLC, Pensacola, FL; 1) measuring the uniaxial acceleration in vertical plane. They were placed on the child's right hip by a plastic clip. The accelerometers were set to record movement in 15-s intervals. Children were informed before each activity period about the upcoming behaviors in which they were to engage, and further instruction about accelerometer use was given such as, not taking off or manipulating the device with extreme movements to avoid spurious data. During each activity period at least one researcher was observing the children to ensure they behaved according the prescribed data collection protocol.

The measurements took place at the children's home or at neighbors'/friends' homes to replicate the natural sedentary behavior environment for children. In the measurement setting, we ensured a consistency through all measurements by making sure that a table to perform nonelectronic sedentary games, the television and the laptop were available. For the outdoor playing, the closest playground in the area was selected. Children were given instructions about staying seated during the sedentary behaviors as well as not touching the accelerometer through the whole measurement process. When children moved around and did not remain seated during the sedentary behaviors they were asked to sit down again and continue with watching TV, playing a computer, or playing a board game. Active minutes were noted in a systematic observation protocol. 


\section{Data Analysis}

Data reduction from accelerometers (each activity lasted $20 \mathrm{~min}$ ) were performed manually with Actilife software, and then transferred to SPSS. The first three minutes of the activity periods were removed, because children needed some time to settle. Figures were created to depict how different cut-points compared with the observed 25th, 50th and 75th percentiles for the cpm derived from each activity.

Table 1 shows the characteristics of four published cut-points $(\leq 100 \mathrm{cpm}$; $\leq 300 \mathrm{cpm}$; $\leq 800 \mathrm{cpm}$; and $\leq 1100 \mathrm{cpm}$ ) that were compared against the observed cut-points for sedentary behaviors $(14,15,18,21)$.

Table 1 Characteristics of Included Calibration Studies to Derive Sedentary Cut-Points for Actigraph Accelerometry Data

\begin{tabular}{|c|c|c|c|}
\hline Study & Participants & Calibration Method & $\begin{array}{c}\text { Sedentary cut } \\
\text { point per minute }\end{array}$ \\
\hline \multirow{5}{*}{$\begin{array}{l}\text { Reilly et al. } \\
(2003)\end{array}$} & $N=30$ & Direct observation & 1100 \\
\hline & 20 boys, 10 girls & Actigraph model 7164 & \\
\hline & $3-4 y$ & Epoch $=1 \mathrm{~min}$ & \\
\hline & & $\begin{array}{l}\text { Activities= Free-living } \\
\text { activities }\end{array}$ & \\
\hline & & $\begin{array}{l}\text { Observation period- } 100 \mathrm{~m} \\
( \pm 17 \text { min })\end{array}$ & \\
\hline \multirow{5}{*}{$\begin{array}{l}\text { Puyau et al. } \\
(2002)\end{array}$} & $N=26$ & Whole room calorimetry & 800 \\
\hline & 12 girls, 14 boys & Actigraph model 7164 & \\
\hline & $6-16$ y & Epoch= $1 \mathrm{~min}$ & \\
\hline & & $\begin{array}{l}\text { Activities= Computer } \\
\text { gaming, drawing, playing } \\
\text { nonexercise sedentary games }\end{array}$ & \\
\hline & & Each activity for $20 \mathrm{~min}$ & \\
\hline \multirow{4}{*}{$\begin{array}{l}\text { Stone et al. } \\
\text { (2009) }\end{array}$} & $N=52$ & Laboratory based & 300 \\
\hline & Only boys & $\begin{array}{l}\text { Actigraph model not speci- } \\
\text { fied }\end{array}$ & \\
\hline & $8-10 y$ & Epoch $=2 \mathrm{~s}$ & \\
\hline & & $\begin{array}{l}\text { Activities= Standing still for } \\
1 \mathrm{~min}\end{array}$ & \\
\hline \multirow{5}{*}{$\begin{array}{l}\text { Treuth et al. } \\
\text { (2004) }\end{array}$} & $N=74$ & Direct calorimetry & 100 \\
\hline & Only girls & Actigraph model 7164 & \\
\hline & $13-14 y$ & Epoch $=30 \mathrm{~s}$ & \\
\hline & & $\begin{array}{l}\text { Activities= Resting, watch- } \\
\text { ing TV, playing computer } \\
\text { games }\end{array}$ & \\
\hline & & Each activity for $7 \mathrm{~min}$ & \\
\hline
\end{tabular}




\section{Results}

In total, 25 children (six boys and 19 girls) aged 5-11 years old were included in the analysis. Counts per minute were assessed in an a priori specified activity. Figures 1-3 show the median, 25th and 75th percentiles of cpm during the different sedentary behaviorss compared with two of the published cut-points $(\leq 100, \leq 300)$. As the cpm for all of the sedentary behaviors were well below $800 \mathrm{cpm}$, only the lower published cut-points are shown in Figures 1-3. Figure 4 shows the median, 25th and 75th percentiles of cpm during outside play compared with the four published sedentary cut-points $(\leq 100, \leq 300, \leq 800, \leq 1,100)$. Figure 1 shows that during computer gaming the 25 th, median and the 75 th cpm percentiles were below all published cut-points. It can also be seen in Figure 1 that the median and the 25 th percentile values were zero for all the minutes. In Figures 2 and 3 (non electronic sedentary games and TV watching) the 25 th percentile values were zero for all the minutes.

Figure 2 illustrates that although the median cpm were below all cut-points during nonelectronic sedentary games, in the second half of the observation period the 75 th percentile values were above $100 \mathrm{cpm}$. However, the $25 \mathrm{th}$, median and the 75th percentile cpm were far below Reilly's and Puyau's cut-points of $\leq 1,100$ and $\leq 800$ respectively.

Figure 3 shows that the median counts during TV watching were below all cut-points. The 75th percentile values of the cpm during this activity were below all three cut-points; however, on some occasions children exceeded $100 \mathrm{cpm}$. Except for the 1100 cut off point of Reilly et al. (10) the median cpm during playing outside activity was above all cut-points. In addition, the 25 th percentile values during playing outside were sometimes even below the 300 and $800 \mathrm{cpm}$ (Figure 4).

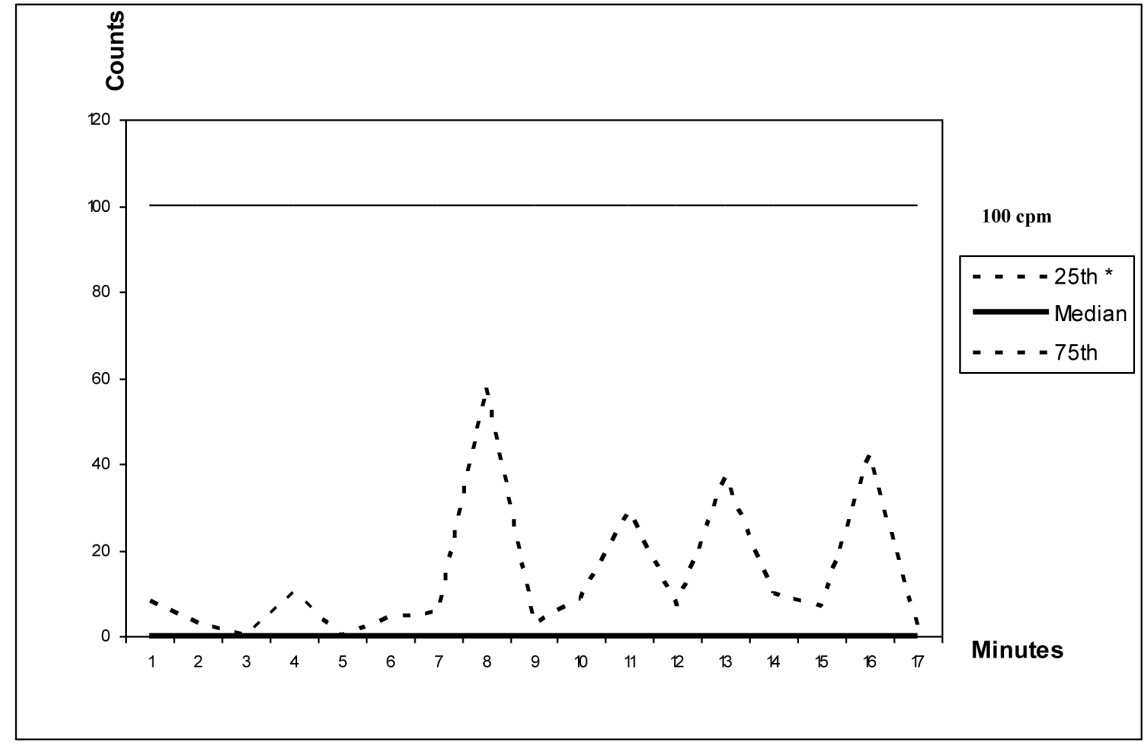

$* 25^{\text {th }}$ percentile $=0$

Figure 1 - The median, 25th and 75th percentiles of counts per minute during computer (PC) gaming. 


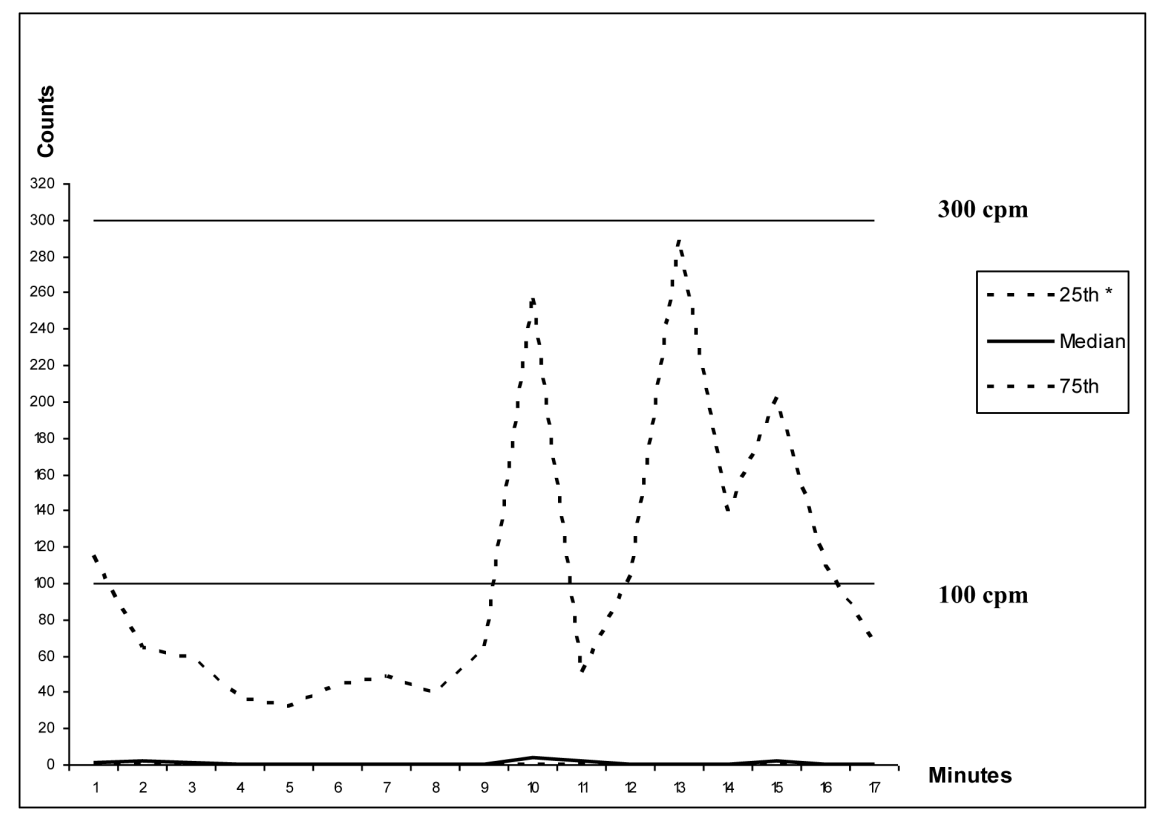

\section{$* 25^{\text {th }}$ percentile $=0$}

Figure 2 - The median, 25th and 75th percentiles of counts per minute during nonelectronic sedentary games.

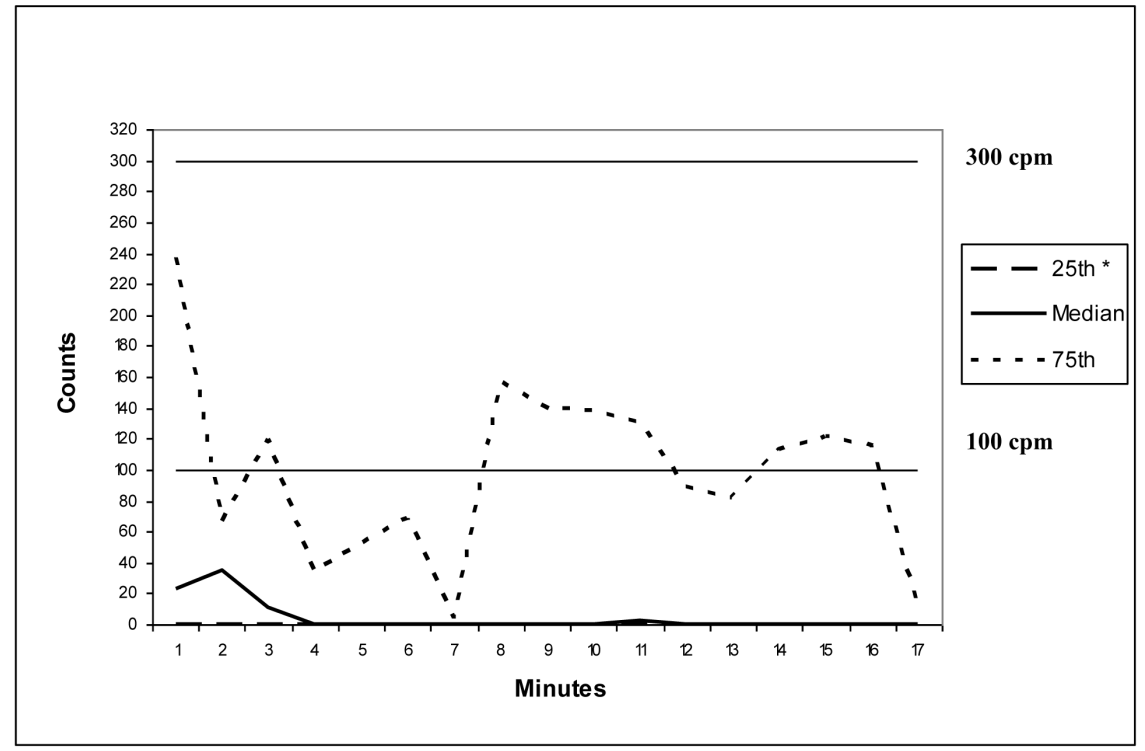

$* 25^{\text {th }}$ percentile $=0$

Figure 3 - The median, 25th and 75th percentiles of counts per minute during TV watching. 


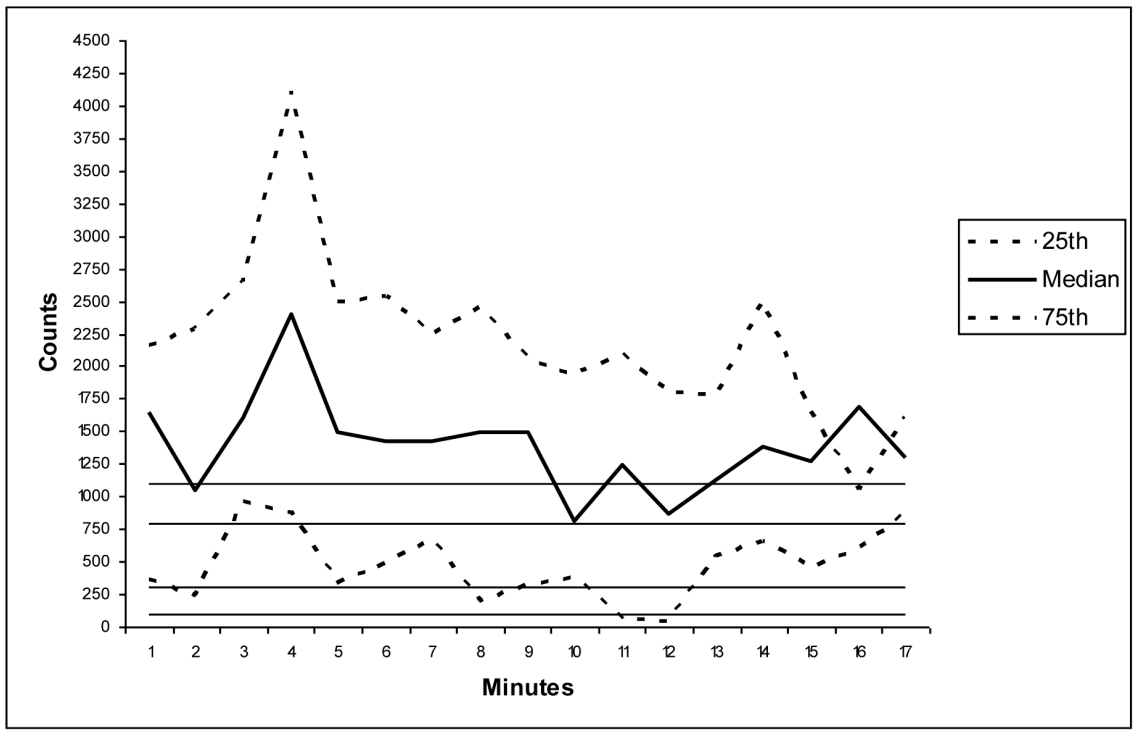

Figure 4 - The median, 25th and 75th percentiles of counts per minute during playing outside.

\section{Discussion}

The aim of the current study was to compare different published accelerometer cutpoints against specific observed sedentary behaviorss among children. The results suggest that estimated time spent in sedentary behaviors would differ considerably using the various published cut-points available. To be able to determine the relationship between health and sedentary behaviors accurate assessment of sedentary time is needed. Selecting different cut-points results in considerable disparity in estimates of time spent sedentary. Furthermore, the application of different cutpoints for assessing children's sedentary time using the Actigraph accelerometer hampers the comparability of studies.

Compared with our own observations of the children's sedentary behaviors we conclude that the cut-point of $1100 \mathrm{cpm} \mathrm{(15)} \mathrm{and} 800 \mathrm{cpm} \mathrm{(14)} \mathrm{are} \mathrm{too} \mathrm{high} \mathrm{and}$ these would lead to an overestimation of the actual time spent sedentary. During TV watching and nonelectronic sedentary games, according to the 75th percentile values the lowest cut-point of $100 \mathrm{cpm} \mathrm{(21)} \mathrm{would} \mathrm{be} \mathrm{too} \mathrm{low;} \mathrm{however,} \mathrm{on} \mathrm{bal-}$ ance, the median cpm values of the three observed sedentary behaviors suggest that $\leq 100 \mathrm{cpm}$ would provide the best estimate of children's sedentary time. The median cpm during playing outside was above all sedentary cut-points apart from those of Reilly's (15), with some 25th percentile values below the 300 and $800 \mathrm{cpm}$. This may be because children were not prompted to engage in continuous physical activity in the outdoor play protocol and is therefore a reflection of children's typical intermittent activity patterns.

In the current study data were collected using a 15-s epoch, while the previous calibration studies used an epoch length ranging from $2 \mathrm{~s}$ to $1 \mathrm{~min}$. A recent study from 
Ojiambo et al. (13) among children with a mean age of 7 years ( \pm 2 years) showed that collecting data with different epoch lengths significantly influenced the estimates of time spent in moderate-to-vigorous physical activity (MVPA) and being sedentary. Using shorter epochs of 15-s compared with 1-min resulted in less sedentary time and higher MVPA time in youth. Considering these findings, one might expect an influence of an epoch length in the calibration studies. However, we cannot further elaborate on this topic due to the absence of comparison of different epoch lengths in a single calibration method. There are also variations in the selected reference activities in the previous calibration studies. In the current study we selected the most common forms of children's sedentary behavior to represent typical daily sedentary behaviors for this age group.

Due to the low sample size we could not examine possible gender or age differences in cut-points. This issue deserves further empirical study. We observed recognizably higher cpm in the beginning of the 20 -min observation time while children were watching TV or playing computer games. Perhaps due to the Hawthorne effects of being observed and the novelty of the experimental condition, children need some time to calm down when starting to watch TV or play computer games. For this reason we excluded the first three minutes of observation time to properly reflect the true sedentary period. However, strengths of our study which should be acknowledged are the use of popular forms of sedentary behavior among children performed in a naturalistic environment.

Further research should extend the assessment of a wider variety of children's sedentary patterns in larger samples that are evenly distributed between boys and girls. To assess the reliability of the observations, more time points of the same activities in the same children assessed by a standard observation tool is recommended. In conclusion, it is important to be aware of the consequences of using the different cut-points when assessing sedentary behaviors in children. Based on our results, the relatively lower cut-point of $100 \mathrm{cpm} \mathrm{(21)} \mathrm{provides} \mathrm{the} \mathrm{most} \mathrm{real-}$ istic estimate of the time children spend sedentary (at least for the three common leisure-time sedentary behaviors assessed in the current study). However, at the individual level there will always be variability and overcome this accelerometer data from each child would need to be individually calibrated, which is not practical in population research. Our findings suggest that the cut-point of $100 \mathrm{cpm}$ will be suitable for children on average across a sample, and is best used in population research for that purpose, rather than at the individual level.

\section{Acknowledgments}

We thank the children and their parents for their cooperation and participation in this study. JS is supported by a National Heart Foundation of Australia and sanofi-aventis Career Development Award. The current address of the first author (CF) is Academic Medical Center, University of Amsterdam Department of Public Health Amsterdam, The Netherlands.

\section{References}

1. ActiGraph. ActiTrainer Research Model. http://www.theactigraph.com/ accessed 201006-04.

2. Ainsworth, B.E., W.L. Haskell, M.C. Whitt, et al. Compendium of physical activities: an update of activity codes and MET intensities. Med. Sci. Sports Exerc. 32:498-514, 2000. 
3. Alhassan, S., J.R. Sirard, and T.N. Robinson. The effects of increasing outdoor play time on physical activity in Latino preschool children. Int. J. Pediatr. Obes. 2:153-158, 2007.

4. Bryant, M.J., J.C. Lucove, K.R. Evenson, et al. Measurement of television viewing in children and adolescents: a systematic review. Obes. Rev. 8:197-209, 2007.

5. Carson, V., I. Janssen. Volume, patterns, and types of sedentary behavior and cardiometabolic health in children and adolescents: a cross-sectional study. BMC Pub Health 4;11:274, 2011.

6. ChinAPaw, M. J. M., K.I. Proper, J. Brug, W. Van Mechelen, A.S. Singh. Relationship between young peoples' sedentary _ehavior and biomedical health indicators: a systematic review of prospective studies. 12; 7:621-632, 2011.

7. Crouter, S.E., K.G. Clowers, and D.R. Bassett. A novel method for using accelerometer data to predict energy expenditure. J. Appl. Physiol. 100:1324-1331, 2006.

8. Hill, J.O., and J.C. Peters. Environmental contribution to the obesity epidemic. Science. 280:1371-1374, 1998.

9. Lubans, D.R., K. Hesketh, D.P. Cliff, et al. A systematic review of the validity and reliability of sedentary _ehavior measures used with children and adolescents. Obes. Rev., 2011. .

10. Marshall, S.J., T. Gorely, and S.J.H. Biddle. A descriptive epidemiology of screen-based media use in youth: a review and critique. J. Adolesc. 29:333-349, 2006.

11. Martínez-Gómez, D., J.C. Eisenmann, S. Gómez-Martínez, A. Veses, A. Marcos, and O.L. Veiga. Sedentary behavior, adiposity and cardiovascular risk factors in adolescents. The AFINOS study. Rev. Esp. Cardiol. 63(3):277-285, 2010.

12. Matthews, C.E., K.Y. Chen, P.S. Freedson, et al. Amount of time spent in sedentary behaviours in the United States, 2003-2004. Am. J. Epidemiol. 167:875-881, 2008.

13. Ojiambo. R., R. Cuthill, H. Budd, et al. Impact of methodological decisions on accelerometer outcome variables in young children. Int J Obes (Lond). 35 Suppl. 1:S98-103, 2011.

14. Puyau, M.R., A.L. Adolph, F.A. Vohra, et al. Validation and calibration of activity monitors in children. Obes. Res. 10:150-157, 2002.

15. Reilly, J.J., J. Coyle, L.A. Kelly, et al. An objective method for measurement of sedentary behaviour in 3-4 year-olds. Obes. Res. 11:1155-1158, 2003.

16. Reilly, J.J., V. Penpraze, J. Hislop, et al. Objective measurement of physical activity and sedentary behaviour: review with new data. Arch. Dis. Child. 93:614-619, 2008.

17. Sardinha, L.B., L.B. Andersen, S.A. Anderssen, et al. Objectively measured time spent sedentary is associated with insulin resistance independent of overall and central body fat in 9- to 10-year-old Portuguese children. Diabetes Care. 31:569-575, 2008.

18. Stone, M.R., A.R. Rowlands, and R.G. Eston. Relationships between accelerometerassessed physical activity and health in children: impact of the activity-intensity classification method. J Sports Sci Med. 8:136-143, 2009.

19. Te Velde, S.J, I, I. Bourdeaudhuij de, M. Thorsdottir, et al. Patterns in sedentary and exercise behaviors and associations with overweight in 9-14-year-old boys and girls-a cross-sectional study. BMC.Public Health 7:16, 2007.

20. Tremblay, M.S., R.C. Colley, T.J. Saunders, G.N. Healy, and N. Owen. Physiological and health implications of a sedentary lifestyle. Appl. Physiol. Nutr. Metab. 35(6):725-740, 2010.

21. Treuth, M.S., K. Schmitz, D.J. Catellier, et al. Defining accelerometer thresholds for activity intensities in adolescent girls. Med. Sci. Sports Exerc. 36:1259-1266, 2004.

22. Wang, Y., and T. Lobstein. Worldwide trends in childhood overweight and obesity. Int. J. Pediatr. Obes. 1:11-25, 2006.

23. Welk, G.J. Principles of design and analyses for the calibration of accelerometry-based activity monitors. Med. Sci. Sports Exerc. 37:501-511, 2005. 